\title{
Age Dependent Accumulation of Spontaneous DNA Damage in Liver of Transgenic Mice over Expressing Ribosomal Protein S3
}

\section{Vijay Hegde ${ }^{1 *}$, Sridevi Yadavilli² and Walter A Deutsch ${ }^{2}$}

${ }^{1}$ Department of Nutritional Sciences, Texas Tech University, 1301 Akron Avenue, Lubbock, Texas 79409, USA

${ }^{2}$ Card Children's National Health System, Center for genetic medicine research, 111 Michigan Ave NW, Washington DC 20010, USA

\begin{abstract}
Oxidative stress has been associated with the aging process and increased cancer incidence mainly due to accumulation of free radical dependent cellular damage. Reactive oxygen species (ROS) induced formation of 7,8-dihydro-8-oxoguanine (8-oxodG) has the potential to mispair with Adenine during DNA replication in key genes involved in the development of cancer. Human ribosomal protein S3 (RPS3) participates in a variety of reactions beyond its role in protein synthesis, such as processing of oxidative DNA damage. We have shown ex vivo that oxidative stress causes RPS3 to translocate to the nucleus, bind to sites of 8-oxodG with an extraordinarily high binding affinity and interferes with 8-oxodG repair in vitro. Furthermore, our in vivo studies using RPS3 over expressing transgenic mice showed translocation of RPS3 to the nucleus in mouse embryonic fibroblasts (MEFs) and co-localization to 8-oxodG foci with an increase in DNA damage. In this study, we show age dependent accumulation of DNA damage (double strand breaks [DSBs] and fragmented DNA) in aging (15-month old) transgenic mice over expressing RPS3. We also observed increase in DNA damage particularly 8-oxodG, in the liver of older transgenic mice which showed a pre-neoplastic pathology and presence of hepatic tumors. The older transgenic livers had significantly higher p53 expression compared to age matched wild-type mice. These results provide evidence for role of RPS3 over expression interfering with DNA repair and predisposing animals to carcinogenesis but also underscore the role of RPS3 expression plays in the DNA damage signaling pathway and in maintenance of genome integrity by channeling cells either towards cell survival (DNA repair) or cell death (apoptosis).
\end{abstract}

Keywords: Ribosomal Protein S3; Oxidative DNA Damage; Aging; Transgenic mice

\section{Introduction}

In living cells reactive oxygen species (ROS) have important physiological functions and are formed continuously as a consequence of metabolic and other biochemical reactions as well as from external factors. Antioxidant defense systems cannot provide complete protection from noxious effects of ROS. As a result, the genome of eukaryotic cells is under continuous attack from a variety of DNA damaging ROS, leading to many types of DNA lesions, which include single-strand breaks (SSBs), double-strand breaks (DSBs), mismatches, chemical modifications of bases or sugars and inter- or intra-strand cross-links [1-4]. Considerable evidence exists indicating that oxidation of nucleic acids can play a causal role in the development of cancer [5]. A common oxidative stress induced DNA base modification, 7 , 8-dihydro-8-oxoguanine (8-oxodG), is found to be the most prevalent pro-mutagenic lesion of guanine [6]. In mammalian cells spontaneous oxidative DNA damage lesions are formed at high rates even in the absence of exogenous DNA damaging agents. In a human cell, it is estimated that 100-500 8-oxodG lesions are formed per day that accumulate with age. There is evidence of multifold increase in cancer rates in some of the tissues in which 8-oxodG accumulates $[7,8]$. High levels of 8-oxodG have been found in human renal carcinomas [9] and in breast carcinoma cells [10] relative to respective normal tissue.

We have previously shown in vitro that RPS3 binds to 8-oxodG with a very high affinity and interfere with its repair $[11,12]$. Mouse embryonic fibroblasts (MEFs) isolated from transgenic mice over expressing RPS3 showed translocation of RPS3 from the cytoplasm to the nucleus and colocalization to 8-oxodG foci. Cells exposed to oxidative stress also showed increased accumulation of DNA damage when compared to MEFs from wild-type mice [13]. However, little is known about the role of RPS3 in inducing tumors due to age dependent accumulation of spontaneous DNA damage. In this study, we evaluated the age-dependent accumulation of spontaneously occurring endogenous oxidative DNA damage (DSBs, fragmented DNA and 8-oxodG), in 15-month old transgenic mice over expressing RPS3 compared with age matched wild-type mice. Comet assay revealed that transgenic over expression of RPS3 increased the agedependent accumulation of DSBs/fragmented DNA in the animal. Liver of aging transgenic mice showed more 8-oxodG and co-localization with RPS 3 in the nucleus. Furthermore, as mice age, over expression of RPS3 predisposed these animals to tumorigenesis, which could be a direct consequence of block to the repair of 8-oxodG by RPS3.

\section{Materials and Methods}

\section{Animals}

RPS3 overexpressing transgenic [13] and wild type C57BL6 mice were maintained on a $12 \mathrm{~h}$ light/dark cycle and were fed a standard mouse lab chow and water ad libitum. 15-month and 4-month old transgenic and wild-type male mice were sacrificed by $\mathrm{CO}_{2}$ asphyxiation and cervical dislocation. Sacrificed animals were necropsied within 5 min of death and entire animals were fixed in $10 \%$ buffered formalin for histopathological examination. For molecular analysis, tissues were collected before fixation and were flash frozen in liquid nitrogen and stored at $-70^{\circ} \mathrm{C}$. Animal protocols were approved by the IACUC for Pennington Biomedical Research Center.

\section{Evaluation of DNA damage in whole blood by the neutral comet Assay}

The alkaline single cell gel electrophoresis assay (comet assay) was

${ }^{*}$ Corresponding author: Vijay Hegde, Department of Nutritional Sciences, Texas Tech University, 1301 Akron Avenue, Lubbock, Texas 79409, Tel: +1 806834 6695; Fax: +1 806742 2926; E-mail: vijay.hegde@ttu.edu

Received May 04, 2016; Accepted May 10, 2016; Published May 17, 2016

Citation: Hegde V, Yadavilli S, Deutsch WA (2016) Age Dependent Accumulation of Spontaneous DNA Damage in Liver of Transgenic Mice over Expressing Ribosomal Protein S3. Mol Biol 5: 164. doi:10.4172/2168-9547.1000164

Copyright: $\odot 2016$ Hegde V, et al. This is an open-access article distributed under the terms of the Creative Commons Attribution License, which permits unrestricted use, distribution, and reproduction in any medium, provided the original author and source are credited. 
performed using a commercially available kit (Trevigen, Gaithersburg, MD). Whole blood $(2 \mu \mathrm{l})$ from 15 -month $(n=3)$ and 4-month old $(\mathrm{n}=3)$ wild-type and RPS3 transgenic mice were collected by tail snip prior to the animals being sacrificed. Aliquots of blood collected were washed twice with $1 \mathrm{ml}$ of PBS and re-suspended in $150 \mu \mathrm{l}$ of $0.5 \%$ low melting agarose (Fisher) and an aliquot of this suspension $(75 \mu \mathrm{l})$ was immediately layered onto comet slides (Trevigen). After the agarose gels were allowed to solidify at $4^{\circ} \mathrm{C}$ for $10 \mathrm{~min}$, slides were placed in prechilled lysis solution (Trevigen) and incubated in the dark for $55 \mathrm{~min}$ at $4^{\circ} \mathrm{C}$. The slides were rinsed twice with TBE and gels were subjected to electrophoresis in TBE for $10 \mathrm{~min}$ at 17 volts. Thereafter, gels were fixed by placing them in ice-cold methanol for $5 \mathrm{~min}$, followed by ethanol for additional $5 \mathrm{~min}$. Gels were then stained with 501 SYBR green dye (Trevigen, 1:10,000) and viewed under a fluorescence microscope (Zeiss Axiophot research microscope) using a FITC filter. Images of 25 randomly selected fields from each gel were acquired using a SPOT-RT slider digital camera and SPOT software (Diagnostic Instruments, Inc).

The extent of DNA damage was determined by calculating the comet moment, which is the integrated density of the comet tail multiplied by the distance from the center of the nucleus to the center of mass of the tail. Computations scored 25 cells for each sample using a macro available from Herbert M. Geller at http://www2.umdnj. $\mathrm{edu} / \sim$ geller/lab/comet-Scoring-Macro.txt. As the rapidly changing intensities of the individual cells are difficult to control, a large variance within each experiment is an unavoidable consequence of using this method. Therefore, a normalizing and variance-stabilizing logarithmic transformation was then applied to the calculated tail moments [1417]. Cell frequency was determined by a visual scoring method [14] classifying DNA damage into the following categories: type 1 - intact nucleus, smooth outer edges; type 2 - intact nucleus, small amount of tailing; type 3 - intact nucleus, large amount of tailing; type 4 shrinking nucleus, large amount of tailing.

\section{Detection of protein expression by western blot analysis}

Total protein from tissues was extracted as previously described [13] and protein concentration determined by Bradford assay [18]. Western blot analysis was performed by resolving the total protein from tissues by SDS-PAGE and transferred onto nitrocellulose membrane by electro transfer technique. Membranes were then blocked in $5 \%$ non-fat dairy milk and immunoblotted with anti-RPS3 (custommade by Proteintech Group, Chicago, IL), anti-p53 (Cell Signaling Technology), anti-H2AX (Cell Signaling Technology), and anti-p21 (Santacruz Biotechnology). All protein bands were normalized with anti-GAPDH (Chemicon International, Temecula, CA). After treating the membranes with appropriate secondary antibodies, antigen bands were visualized by enhanced chemiluminescence technique using western lightning chemiluminescence reagent plus (Perkin-Elmer). Densitometric tracing of the protein bands was performed by using AlphaEaseFc software (Alpha Innotech Corporation). Protein from tissues was extracted from 3 differenyt mice for each group.

\section{Histopathology of tissue sections}

Preparation of slides for histopathology evaluation was done at the Research Animal Diagnostic Laboratory (RADIL) in Columbia, MO. Briefly, formalin fixed tissues were trimmed, embedded in paraffin, sectioned to a thickness of 5 to $6 \mu \mathrm{m}$, stained with $\mathrm{H} \& \mathrm{E}$ and examined with light microscope. 10 individual mice were examined for each group.

Detection of RPS3 and 8-oxodG for expression and colocalization analysis by immunofluorescence microscopy

Formalin fixed liver and tumor tissues from 15-month old wild- type and RPS3 over expressing transgenic mice were used to detect RPS3/8-oxodG expression and co-localization. Slides were first deparaffinized by incubating in xylenes for $10 \mathrm{~min}(2 \mathrm{X})$, in $100 \%$ ethanol for $10 \mathrm{~min}(2 \mathrm{X})$, in $95 \%$ ethanol for $10 \mathrm{~min}(2 \mathrm{X})$, in $70 \%$ ethanol for $10 \mathrm{~min}(1 \mathrm{X})$ and then washed in water for $10 \mathrm{~min}(2 \mathrm{X})$. The slides were then placed in $10 \mathrm{mM}$ sodium citrate antigen retrieval solution and boiled for $20 \mathrm{~min}$. following which the slides were rinsed in water (3X) for $5 \mathrm{~min}$ each and finally rinsed in $1 \mathrm{X}$ PBS for $5 \mathrm{~min}$. After washing in PBS, slides were blocked in $1 \%$ BSA for $1 \mathrm{~h}$ and incubated with anti-RPS3 (Proteintech, IL) and anti-8-oxodG (AntiDNA/RNA oxidative damage markers monoclonal antibody, QED Biosciences Inc) antibodies for an additional hour. Samples were then washed in PBS, and further incubated with rhodamine red-anti-rabbit (Molecular Probes) and Alexa 488-anti-mouse (Invitrogen) secondary antibodies for $1 \mathrm{~h}$. After washing again in PBS, samples were mounted with 4'-6-diamidino-2-phenylindole (DAPI) containing vectashield mounting media. Localization of RPS3, 8-oxoG, and nuclei were performed by fluorescence microscopy using Zeiss Axioplan 2 upright microscope equipped with Photometrics Coolsnap HQ CCD camera. The filter sets used for visualizing the fluorescent signals include DAPI (EX 360/40, EM 460/50), FITC (EX HQ487/25 EM GQ535/40, D Q505lp) and Texas Red (EX 560/55, EM 645/75). Captured images were processed using ImageJ software. Expression intensity was determined as previously described [13], where the microscope parameters where set for control tissue (wild-type) and the transgenic tissue slides were viewed at the same setting.

\section{Measurement of 8-oxodG}

DNA from flash frozen liver tissues of wild-type and RPS3 over expressing transgenic mice were analyzed by the Oxidative Stress core at the University of Texas Health Science Center, San Antonio to measure levels of 8-oxodG as described previously $[19,20]$. Liver tissues from 3 individual mice from each group were used for analysis.

\section{Statistical analysis}

Groups were compared using one-tailed student T-test to determine significance. $\left({ }^{\star} \mathrm{p}<0.05,{ }^{* *} \mathrm{p}<0.005\right)$.

\section{Results}

\section{Effect of RPS3 over expression on age-dependent DNA damage accumulation}

We have previously reported that over expression of RPS3 in oxidatively stressed MEFs results in increase in DNA damage that involves the nuclear translocation of RPS3 to sites of 8-oxodG and the likely blocking of DNA repair at this lesion [13]. We hypothesized that a block to repair of 8-oxodG due to RPS3 over expression will lead to accumulation of spontaneously occurring endogenous oxidative DNA damage as animals age.

To test this hypothesis in vivo, we quantified the accumulation of DNA damage in the live animal. We analyzed tail snip blood prior to sacrifice from 15-month and 4-month old ( $\mathrm{n}=3$ each) transgenic male mice over expressing RPS3 and age-matched wild type controls by the neutral comet assay that exclusively detects double strand breaks (DSBs) or fragmented DNA.

As seen in (Figure 1A), in younger (4-month) animals, the presence or absence of the RPS3 overexpression had very little effect on the calculated tail moment (the integrated density of the comet tail multiplied by the distance from the center of the nucleus to the center of mass of the tail). However, the older (15-month) transgenic 

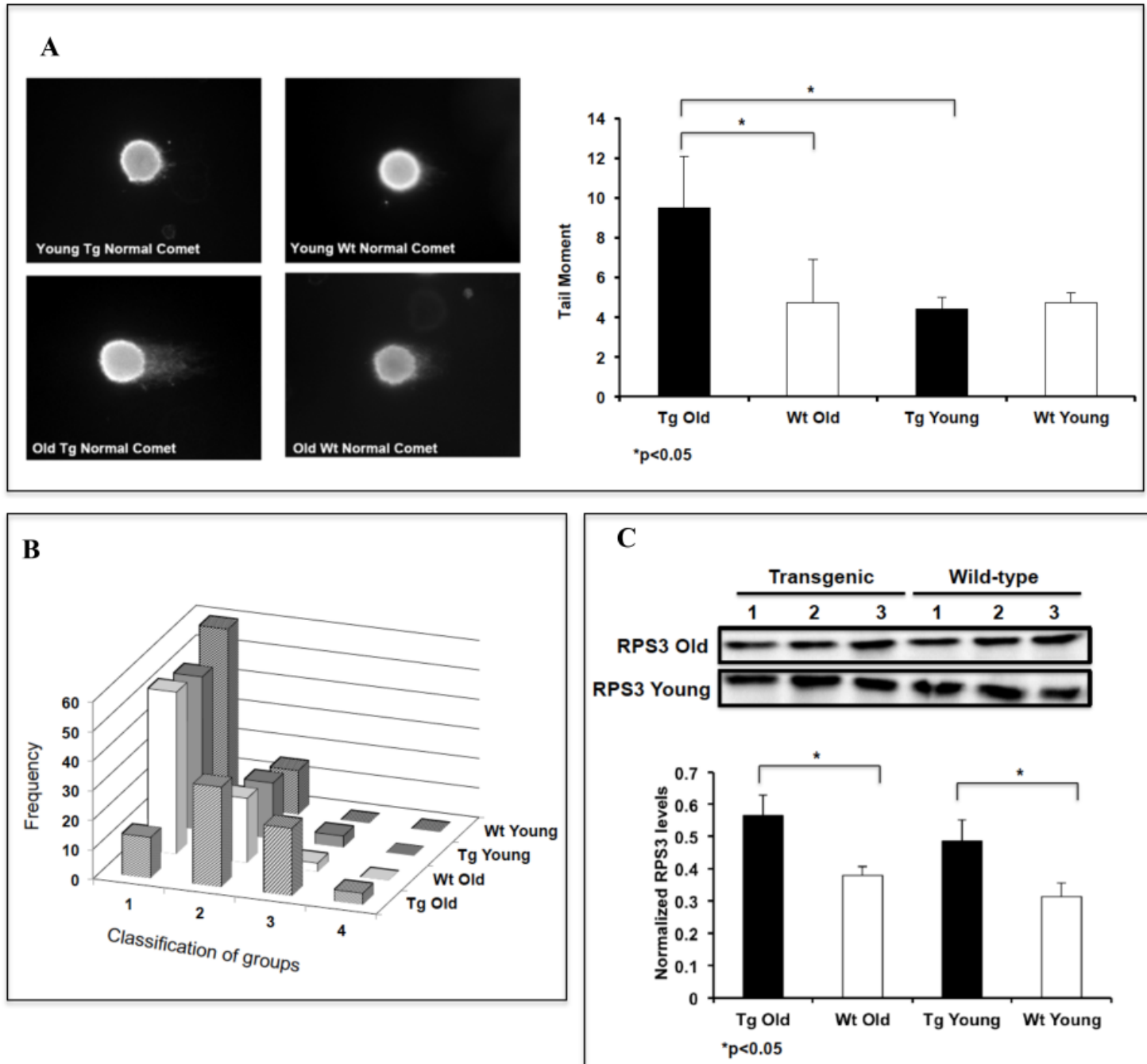

Figure 1: Over expression of RPS3 increases the levels of DNA damage in aging mice. Whole blood from young (4-month) and old (15-month) male mice was evaluated for the extent of DNA damage due to DSBs and/or DNA fragmentation by neutral comet assay. (A) Shows DNA damage expressed as a mean of tail moments from 3 animals for each group. 15-month old RPS3 over expressing transgenic mice show significantly more DNA damage compared with age-matched wild-type mice and young (4-month) transgenic mice. DNA damage to individual cells was also determined by a visual scoring method (as described in methods) to generate the cell frequency graph and values represented with SD. (B) 15-month old transgenic mice show more damaged cells (types 3 and 4 ) compared to age matched wild-type mice similar to that observed with comet tail moment. (C) To determine cellular expression of RPS3, whole cell lysates from liver tissue of 15-month and 4-month transgenic mice and age-matched wild-type controls were analyzed by western blotting. Average RPS 3 expression from 3 replicates normalized to their respective GAPDH expression is shown with SD $(\mathrm{p} \leq 0.05)$.15-month and 4-month old transgenic mice show significantly higher RPS3 expression compared with age-matched wild-type controls.

mice overexpressing RPS3 showed a two-fold increase in DNA damage compared with age-matched wild-type animals. These results would suggest a significantly higher accumulation of DNA damage repair intermediates (DSB's or fragmented DNA) in the older transgenic mice compared to the younger transgenic mice (Figure 1A). DNA damage to individual cells was subsequently conducted using a visual scoring method [14] classifying in this case the extent of DNA damage into the 4 different categories as described in the materials and methods section. Similar to the tail moment data, cell frequency graph shows that older (15-month) transgenic mice have more damaged cells (types 3 and 4) (Figure1B) compared with age matched wild-type mice (types 1 and 2). The younger wild-type and transgenic mice mainly show more undamaged cells (types 1 and 2) (Figure 1B).

To examine any age dependent difference in RPS3 expression, we evaluated RPS3 levels in liver tissues from age matched transgenic and wild-type mice by western blotting using specific RPS3 antibody. As seen in Fig. 1C, RPS3 was significantly over expressed in both the 15-month (Tg old) and 4-month old (Tg young) transgenic mice compared to wild-type controls but there was no difference in over expression levels between the old and young transgenic mice. Similarly when we compared the endogenous RPS3 expression among the wild-type animals, there was a 20\% increase in RPS3 expression with age though not statistically significant.

Collectively, these results indicate that the observed increase in DNA damage (DSBs and/or fragmented DNA) with whole blood in 15-month old transgenic mice can be directly correlated to RPS3 over expression leading to accumulation of DNA damage with age.

RPS3 over expression increases DNA double strand breaks (DSBs) and 8-oxodG levels

As a further means of showing that the increase in DNA damage 
accumulation observed in whole blood was due to the presence of DSBs, we examined the status of phosphorylation of H2AX, a marker for DSB [21]. Pooled protein extracts from liver of RPS3 over expressing or wild-type animals was analyzed by immunoblotting using a specific antibody for phosphorylated H2AX. Expression of phosphorylated $\mathrm{H} 2 \mathrm{AX}$ was determined by normalizing the protein bands relative to cellular GAPDH expression. Pooled extracts were used to better represent the data as expression from individual animals was low. As seen in Figure 2A, there is a $25 \%$ increase in phospho-H2AX (H2AXp) levels in 15-month old transgenic animals compared with age matched wild-type animals and a $40 \%$ increase compared with 4-month old transgenic mice indicating exacerbation of DSB accumulation over age in 15-month old RPS3 over expressing mice.

We then asked the question whether the increase in DNA damage accumulation observed in the liver tissue from these animals was associated by an increase in oxidative damage, particularly 8-oxodG. Measurement of 8-oxodG levels by $\mathrm{Na}$ iodide method to isolate DNA from tissues, revealed that there is a $20 \%$ and $44 \%$ increase in 8 -oxodG levels in the RPS3 over expressing 15-month and 4-month old animals, respectively, compared with age-matched wild-type controls (Figure 2B). This increase in 8-oxodG levels correlates with RPS3 expression, which once again highlights the role of RPS3 over expression in blocking oxidative DNA damage processing.

\section{Over expression of RPS3 predisposes transgenic animals to tumorigenesis}

Histopathological analysis using formalin fixed paraffin embedded (FFPE) sections was performed on 10 male mice each from 15-month and 4-month old transgenic mice and age matched wild-type animals, respectively. Two out of the ten 15-month old F3 transgenic animals
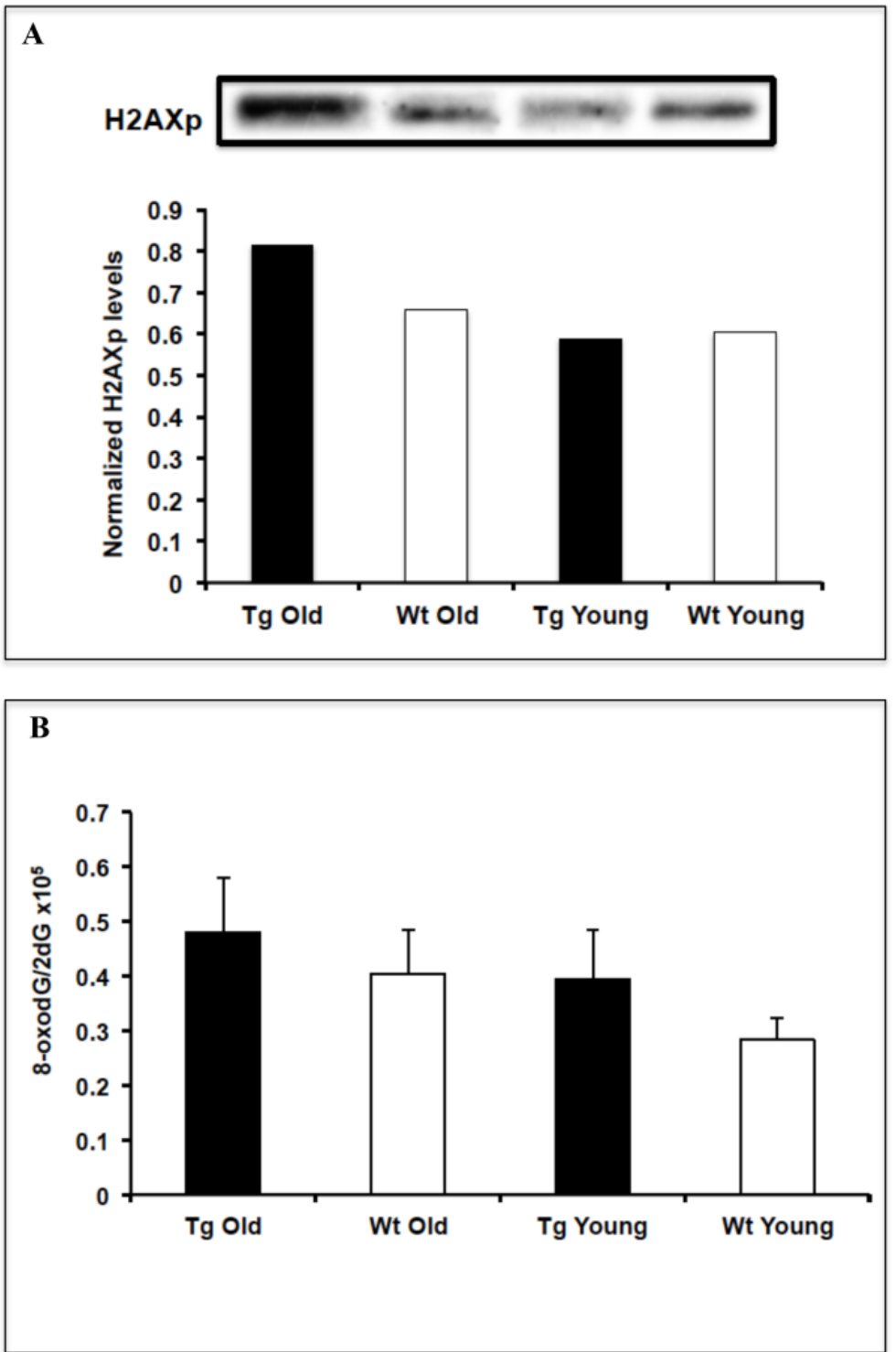

Figure 2: RPS3 over expression increases DNA double strand breaks (DSBs) and 8-oxodG levels (A) H2AXplevels in whole cell lysates of liver tissue from age matched RPS3 over expressing and wild-type mice were determined by western blotting using a specific anti-H2AXp antibody. H2AXp expression normalized to GAPDH is shown in the graph. (B) 8-oxodG levels in liver tissue from RPS3 over expressing and wild-type mice were determined by Na iodide method to isolate DNA from tissues. 
Citation: Hegde V, Yadavilli S, Deutsch WA (2016) Age Dependent Accumulation of Spontaneous DNA Damage in Liver of Transgenic Mice over Expressing Ribosomal Protein S3. Mol Biol 5: 164. doi:10.4172/2168-9547.1000164

Page 5 of 8

showed visible hepatocellular carcinomas (Figure 3A). Additionally, all of the transgenic mice had multifocal proliferation of oval cells in the liver, which were not observed in the wild-type controls. All of the aged transgenic mice and $33 \%$ of the wild type mice had varying degrees of crypt epithelial hyperplasia with mild inflammatory infiltrates in the cecum.

These observations and our previous findings [13] led us to question whether RPS3 over expression and blocking of DNA repair promotes tumorigenesis due to age dependent accumulation of oxidative DNA damage. If this were true, we would expect to see an increase in RPS3 and 8-oxodG expression and co-localization of RPS3 to sites of 8-oxodG in the 15-month old RPS3 over expressing transgenic mice compared with age matched wild-type controls. Further we would expect to see an augmentation in RPS3/8-oxodG expression and co-localization in the tumor tissue from the older transgenic mice compared with normal (non-tumor) tissue.
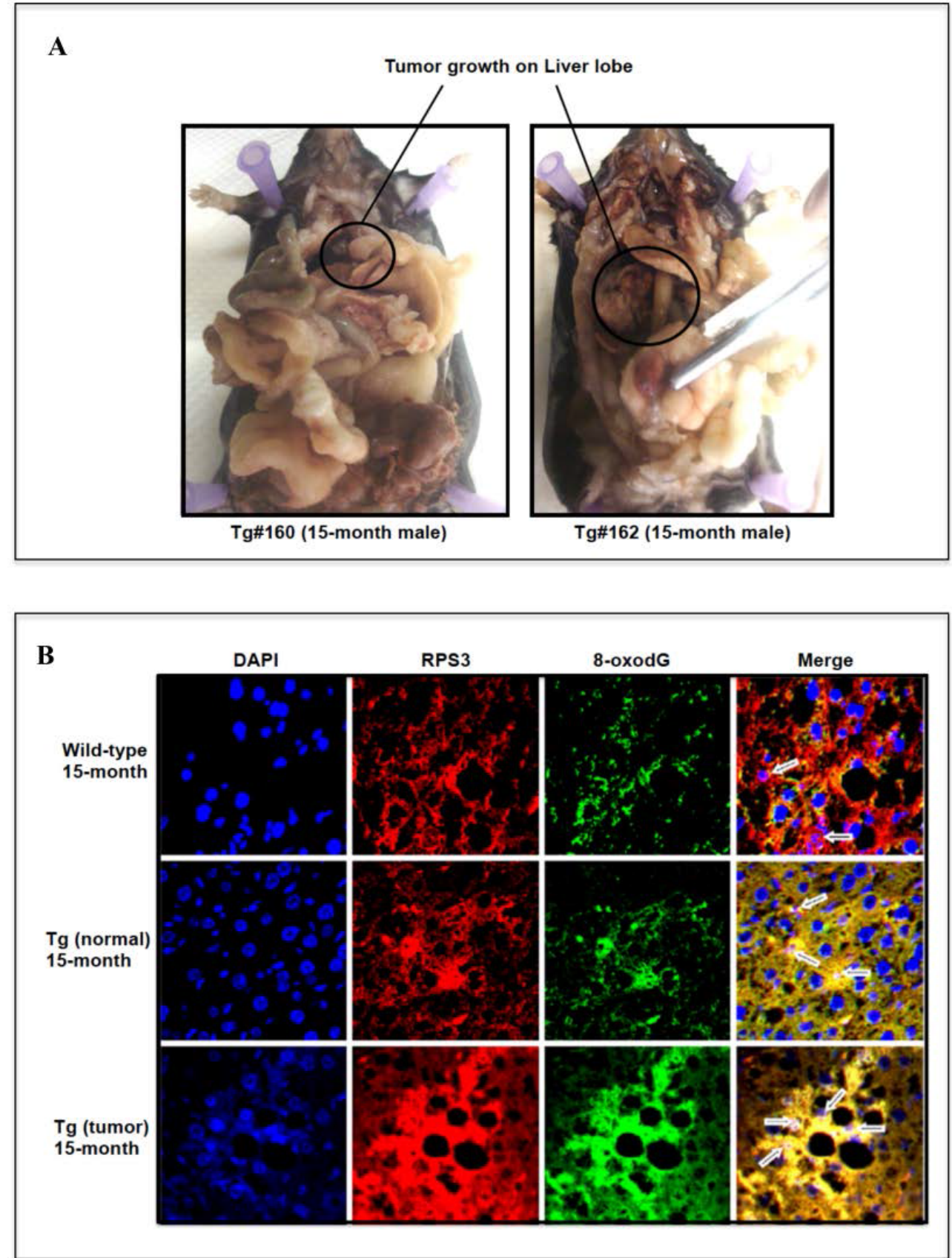

Figure 3: RPS3 over expression leads to DNA damage accumulation and tumorigenesis.

(A) Gross necropsy examination of liver from 15-month old, F3 transgenic mice showed the presence of growth on livers.

(B) Tissue sections from liver of 15-month old wild-type, RPS3 over expressing mice and the tumor sections from older transgenic mice were evaluated by immunoflourescence for detection of RPS3/8-oxodG expression and co-localization. A representative of images obtained with rhodamine (RPS3), FITC (8-oxoG), and UV (nuclei) filters are shown. Additionally, a merge of rhodamine, FITC, and UV images is shown, in which foci of the co-localization of RPS3 and 8-oxoG within the nuclei is indicated by arrows. 
To examine this hypothesis, we performed immunofluorescence analysis for RPS3 and 8-oxodG expression in fixed liver tissue sections from 15-month old wild-type and RPS3 over expressing mice and sections from the hepatocellular carcinoma detected in 15-month old transgenic mice. As seen in Fig.3B, the 15-month old wild-type mice show translocation (pink; merge) of RPS3 (red) into the nucleus (blue) and some co-localization (yellow; merge) of RPS3 with sites of 8-oxodG (green). Arrows in the merge panel for 15-month old transgenic mice show a marked increase in RPS3 translocation to the nucleus and colocalization with sites of 8 -oxodG (yellow; merge). The increase in co-localization observed with 15-month old RPS3 over expressing transgenic mice liver tissue (non-tumor) is further exaggerated in the liver tumor tissue from these mice, where we observe a distinct increase in RPS3 (red) and 8-oxodG (green) expression with a pronounced increase in co-localization (yellow; merge) of RPS3 to sites of 8-oxodG. These observations provide compelling evidence for age-dependent RPS3 over expression, promoting DNA damage accumulation that leads to tumorigenesis.

\section{Effect of RPS3 over expression on hepatic cellular levels of p53}

Histopathological examination of 15-month old F3 transgenic mice showed multifocal proliferation of progenitor oval cells in the liver, which could be a consequence of hepatic cells undergoing apoptosis [22,23]. Our ex vivo data showed that RPS3 interacts with the apoptosis regulatory proteins p53 and MDM2 [24], which led us to hypothesize that accumulation of DNA damage by blocking DNA repair, could channel cells towards apoptosis to maintain genomic integrity [24]. To further test the effect of RPS3 over expression in liver cells, we determined cellular levels of p53 in the 15-month and 4-month transgenic mice compared to age-matched wild-type controls by western blot analysis.

Interestingly, the over expression of RPS 3 in liver (as seen in Figure 1C) resulted in a 2-fold increase in hepatic p53 levels in 15-month old transgenic mice compared to age-matched wild-type mice (Figure $4 \mathrm{~A}$ ). The 4-month old transgenic mice (Tg young) showed a 1.6-fold increase (though not statistically significant) in cellular p53 levels in the liver compared to wild-type (Wt young) control. We also observe a significant increase in cellular p53 expression in the 15-month old transgenic mice over expressing RPS3 compared with 4-month old transgenic mice (Figure 4A). This further emphasizes age-dependent accumulation of oxidative DNA damage for the observed increase in p53 levels, as similar RPS3 expression was seen in both 15-month and 4-month old transgenic mice (Figure 1C). Similarly, we observe a 1.6 fold increase in p53 levels in 15-month old wild-type mice compared with 4-month old wild-type mice (Figure 4A). The observation that over expression of RPS 3 increases cellular p53 levels is also in agreement with our previous ex vivo data, which showed that increasing amounts of RPS3 protected p53 from MDM2 mediated ubiquitination [24].

The impact of increase in $\mathrm{p} 53$ levels on the downstream target genes was evaluated by determining the p21 protein level in the liver cells from transgenic and wild-type animals by western blot analysis. Similar to $\mathrm{p} 53$ levels (Figure $4 \mathrm{~A}$ ) in these cells, we observe a 2.1 fold increase in p21 expression in the 15-month old transgenic animals compared with age matched wild-type mice (Figure $4 \mathrm{~B}$ ) and also a significant increase p21 levels in 15-month old transgenic mice compared with 4-month old transgenic mice (Figure 4B). A similar increase in p21 expression is observed in 4-month old transgenic mice compared with age-matched wild-type mice (Figure 4B). These observations are in agreement that p53 activation in response to stress or DNA damage results in p21 induction mediated $\mathrm{G} 1$ cell cycle arrest $[25,26]$.
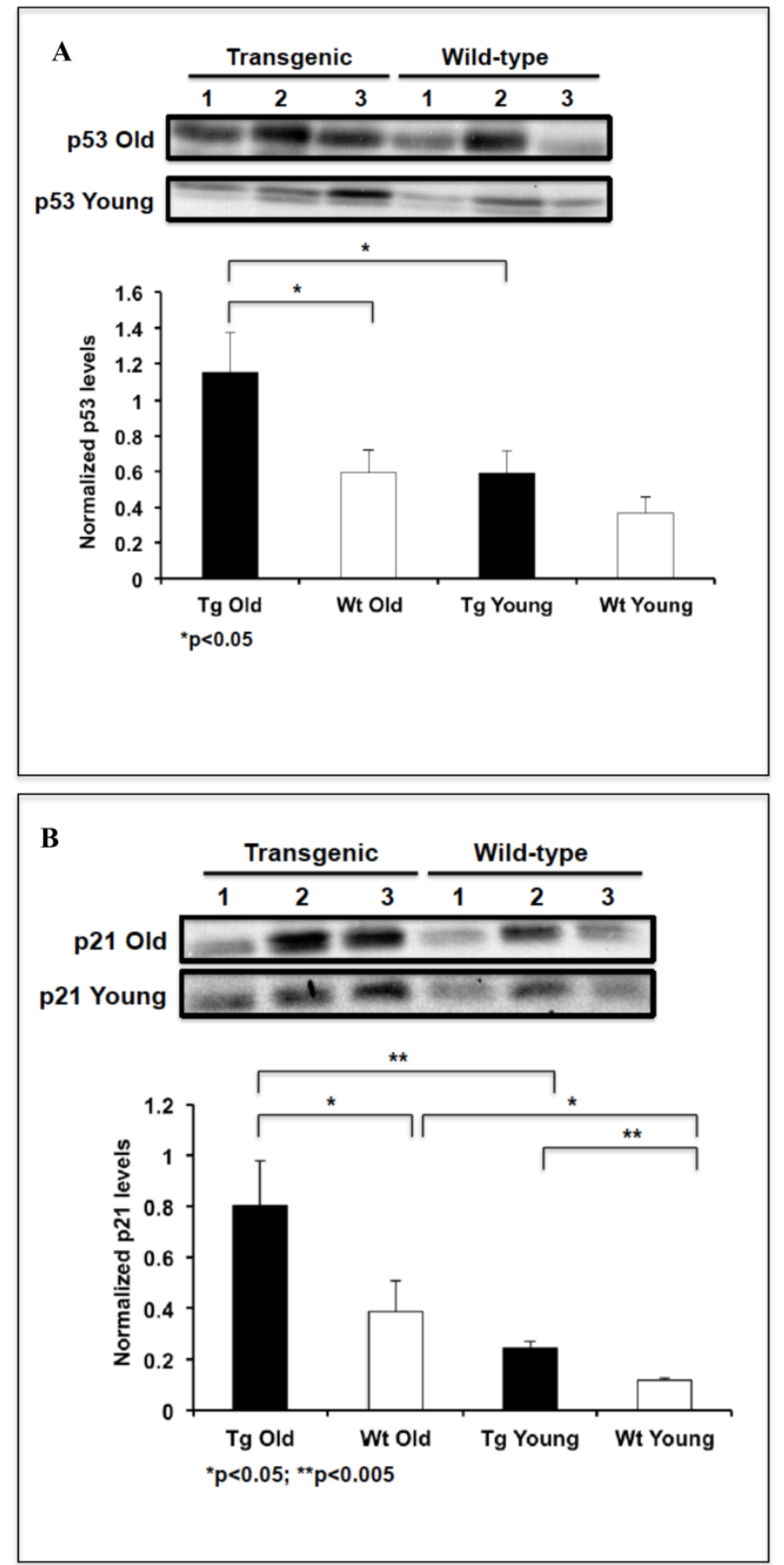

Figure 4: Over expression of RPS3 increases cellular p53 and p21 levels. Whole cell lysates from liver tissue of 15-month and 4-month transgenic mice and age-matched wild-type controls were analyzed by western blotting, utilizing mouse monoclonal anti-p53 and anti-p21 antibodies. Cellular p53 (A) and p21 (B) levels from triplicate samples were normalized by respective GAPDH levels and represented as average of triplicates with SD. RPS3 over expressing 15-month old transgenic mice shows significant increase in cellular p53 and p21 expression in the liver compared with age-matched wild-type control.

\section{Discussion}

Oxidative stress plays a vital role in the initiation, promotion and malignant conversion (progression) stages of carcinogenesis. DNA mutation is a crucial step in carcinogenesis and elevated levels of oxidative DNA lesions are seen in a variety of tumors, strongly implicating such damage in the etiology of cancer. We have previously 
observed that RPS3 possesses a very high apparent binding affinity for 8-oxodG DNA modifications that blocks the liberation of this base by the 8 -oxogunaine DNA glycosylase, OGG1 [11,12]. This implies that a block to 8-oxodG repair would result in accumulation of this modified lesion as the animal ages and RPS3 nuclear over expression will increase this accumulation. Here we show that 15-month old RPS3 over expressing transgenic mice have increased DNA damage compared with age-matched wild-type mice. DNA damage (DSBs or fragmented DNA) as determined by neutral comet assay using whole blood from RPS3 over expressing mice was also significantly higher in the 15-month old transgenic mice compared with younger 4-month old transgenic mice even though we observe similar RPS3 over expression in both transgenic mice. This further highlights our hypothesis that RPS3 over expression and block to DNA repair will result in accumulation of DNA damage in an age-dependent manner.

Older transgenic mice showed an increase in DNA DSBs in the liver tissue as determined by phosphorylation of H2AX as well as an increase in 8-oxodG levels. One possible explanation is that the observed increase in DNA damage by comet assay is mainly DSBs and fragmented DNA, which could be formed as DNA repair intermediates during the processing of 8-oxodG mutations and we have shown that RPS3 blocks DNA repair by hOGG1[12]. RPS3 has binding affinity for 8-oxodG and abasic sites [11], therefore it appears that majority of accumulated oxidative damage over age could remain as repair intermediates.

Pathological examination of liver from transgenic RPS3 over expressing mice showed the presence of visible hepatocellular carcinoma in 2 out of 10, 15-month old transgenic animals and all of the older transgenic animals showed multifocal proliferation of oval cells, which was not observed in the age-matched wild-type animals. In adult mice, oval cells proliferate in response to hepatic inflammation as well as in response to hepatocyte loss through chemical injury, apoptosis or partial hepatectomy $[22,23]$. This is suggestive that RPS3 transgenic mice are undergoing increased levels of apoptosis, which is indicative of a pre-neoplastic condition [27]. Our results show increased and wide spread RPS3 co-localization with foci of 8-oxodG in the nucleus of the normal as well as tumorous liver tissue from older transgenic animals as compared to normal liver tissue from wild-type. This observation provides convincing evidence linking RPS3 co-localization to sites of 8-oxodG and blocking its repair, resulting in age dependent accumulation of this lesion and possible DSBs/fragmented DNA, which pre-disposes the animals to tumorigenesis.

The increase in p53 levels observed in wild-type and transgenic older mice (Figure 4) is not surprising as p53 becomes functionally active in response to stress signals and triggers either a transient cell cycle arrest, cell death (apoptosis) or permanent cell cycle arrest (cellular senescence) [28-30]. Increase in p53 supports the histopathological observation of oval cells (apoptotic cells) in the liver of older transgenic animals and increased accumulation of DNA damage observed by comet assay (Figure 1A), which could be a direct consequence of p53 mediated cell death. The increase observed in p21 kinase protein levels (Figure 4B) is known to induce irreversible G1 phase cell cycle arrest to channel cells towards apoptosis [31], once again pointing towards the possibility that the observed pathology and DNA damage accumulation seen in the older RSP3 over expressing animals is due to cellular apoptosis following block to DNA repair. Studies have also shown ageenhanced apoptosis with DNA fragmentation in various types of cells usually associated with higher p53 expression [32].

The activation of p53 in response to cellular stress is to maintain genomic integrity by acting as a tumor suppressor. So the question to be addressed is if the role of $\mathrm{p} 53$ is protective then why do we see tumors or the susceptibility of tumor development in older mice over expressing RPS3. Our previous studies showing interaction and protection of $\mathrm{p} 53$ by RPS3 had led us to hypothesize that accumulation of DNA damage by blocking DNA repair, could channel cells towards apoptosis to maintain genomic integrity [24]. One suggestion that has been put forth is that the binding of RPS3 moderates the extent and timing of the repair of 8-oxodG residues that are in close proximity to one another so as to prevent the formation of cytotoxic double-strand breaks (DSBs). It is possible that as animals age the protection provided by RPS3 decays over time and binding of RPS3 to sites of DNA damage is more of a "non-productive" binding. Based on the observations presented here it seems likely that the 15-month old RPS3 over expressing mice are accumulating more DSBs in the liver along with DNA fragmentation and a large percentage of liver cells are undergoing p53 mediated apoptosis. A combination of all these events could be causing genomic instability leading to tumorigenesis.

It has been reported that the production of many ribosomal proteins is up regulated in several cancers [33] and additionally RPS3 has been shown to be radio-resistant or involved in invasion of tumor cells $[34,35]$. More recently it has been shown that secreted RPS3 expression level is increased in malignant cells [36] and also RPS3 over expression mediates osteosarcoma invasion and metastasis of osteosarcoma [37] Together, these observations emphasize the role of RPS3 expression in promoting malignancy and support our observation.

\section{Conclusions}

In this work we have established a link between RPS3 over expression leading to age dependent accumulation of spontaneous DNA damage and this increase in DNA damage predisposes animals to tumorigenesis. The observations presented here and the recent reports by others draws attention to RPS3 expression being key in the DNA damage-signaling pathway and in maintenance of genome integrity by channeling cells either towards cell survival (DNA repair) or cell death (apoptosis). Future work would involve modulating RPS3 expression to determine its role in maintaining genome integrity and protecting cells from tumorigenesis.

\section{Acknowledgements}

This work utilized the facilities of the Cell Biology and Bioimaging Core that are supported in part by COBRE (NIH P20-RR021945) and CNRU (NIH 1P30DK072476) center grants from the National Institutes of Health. This work was supported in part by a supplement to US Public Health Service grant CA 109798 to WAD. This research was conducted while Vijay Hegde was an AFAR Research grant recipient.

\section{Conflict of Interest Statement}

The authors declare that there are no conflicts of interest.

\section{References}

1. Caldecott $\mathrm{KW}$ (2007) Mammalian single-strand break repair: mechanisms and links with chromatin. DNA Repair (Amst) 6: 443-453.

2. de Boer J, Hoeijmakers JH (2000) Nucleotide excision repair and human syndromes. Carcinogenesis 21: 453-460.

3. Helleday T, Lo J, van Gent DC, Engelward BP (2007) DNA double-strand break repair: from mechanistic understanding to cancer treatment. DNA Repair (Amst) 6: 923-935

4. Kunkel TA, Erie DA (2005) DNA mismatch repair. Annu Rev Biochem 74: 681-710.

5. Poulsen HE (2005) Oxidative DNA modifications. Exp Toxicol Pathol 57 Supp 1: $161-169$.

6. Shibutani S, Takeshita M, Grollman AP (1991) Insertion of specific bases during DNA synthesis past the oxidation-damaged base 8-oxodG. Nature 349: 431434 
Citation: Hegde V, Yadavilli S, Deutsch WA (2016) Age Dependent Accumulation of Spontaneous DNA Damage in Liver of Transgenic Mice over Expressing Ribosomal Protein S3. Mol Biol 5: 164. doi:10.4172/2168-9547.1000164

Page 8 of 8

7. Russo MT, De Luca G, Degan P, Parlanti E, Dogliotti E, et al. (2004) Accumulation of the oxidative base lesion 8-hydroxyguanine in DNA of tumorprone mice defective in both the Myh and Ogg1 DNA glycosylases. Cancer Res 64: 4411-4414.

8. Xie Y, Yang H, Cunanan C, Okamoto K, Shibata D, et al. (2004) Deficiencies in mouse Myh and Ogg1 result in tumor predisposition and $\mathrm{G}$ to T mutations in codon 12 of the K-ras oncogene in lung tumors, Cancer Res, 64 3096-3102.

9. Okamoto K, Toyokuni S, Uchida K, Ogawa O, Takenewa J, et al. (1994) Formation of 8-hydroxy-2'-deoxyguanosine and 4-hydroxy-2-nonenal-modified proteins in human renal-cell carcinoma. Int J Cancer 58: 825-829.

10. Toyokuni S, Okamoto K, Yodoi J, Hiai H (1995) Persistent oxidative stress in cancer. FEBS Lett 358: 1-3.

11. Hegde V, Wang M, Deutsch WA (2004) Characterization of human ribosomal protein S3 binding to 7,8-dihydro-8-oxoguanine and abasic sites by surface plasmon resonance. DNA Repair (Amst) 3: 121-126.

12. Hegde V, Wang M, Mian IS, Spyres L, Deutsch WA (2006) The high binding affinity of human ribosomal protein $\mathrm{S} 3$ to 7,8 -dihydro-8-oxoguanine is abrogated by a single amino acid change. DNA Repair (Amst) 5: 810-815.

13. Hegde V, Yadavilli S, McLaughlin LD, Deutsch WA (2009) DNA repair efficiency in transgenic mice over expressing ribosomal protein S3. Mutat Res 666: 1622.

14. Dellinger B, Pryor WA, Cueto R, Squadrito GL, Hegde V, et al. (2001) Role of free radicals in the toxicity of airborne fine particulate matter. Chem Res Toxicol 14: $1371-1377$

15. Deutsch WA, Kukreja A, Shane B, Hegde V (2001) Phenobarbital, oxazepam and Wyeth 14,643 cause DNA damage as measured by the Comet assay. Mutagenesis 16: 439-442.

16. Heilbronn LK, de Jonge L, Frisard MI, DeLany JP, Larson-Meyer DE, et al. (2006) Effect of 6-month calorie restriction on biomarkers of longevity, metabolic adaptation, and oxidative stress in overweight individuals: a randomized controlled trial. JAMA 295: 1539-1548.

17. Vicente MG, Nurco DJ, Shetty SJ, Osterloh J, Ventre E, et al. (2002) Synthesis, dark toxicity and induction of in vitro DNA photodamage by a tetra(4-nidocarboranylphenyl) porphyrin. J Photochem Photobiol B 68: 123-132.

18. Bradford MM (1976) A rapid and sensitive method for the quantitation of microgram quantities of protein utilizing the principle of protein-dye binding. Anal Biochem 72: 248-254.

19. Hamilton ML, Guo Z, Fuller CD, Van Remmen H, Ward WF, et al. (2001) A reliable assessment of 8-oxo-2-deoxyguanosine levels in nuclear and mitochondrial DNA using the sodium iodide method to isolate DNA. Nucleic Acids Res 29: 2117-2126

20. Hamilton ML, Van Remmen H, Drake JA, Yang H, Guo ZM, et al. (2001) Does oxidative damage to DNA increase with age? Proc Natl Acad Sci U S A 98: 10469-10474.
21. Paull TT, Rogakou EP, Yamazaki V, Kirchgessner CU, Gellert M, et al. (2000) A critical role for histone $\mathrm{H} 2 \mathrm{AX}$ in recruitment of repair factors to nuclear foci after DNA damage. Curr Biol 10: 886-895.

22. Bustos M, Sangro B, Alzuguren P, Gil AG, Ruiz J, et al. (2000) Liver damage using suicide genes. A model for oval cell activation. Am J Pathol 157: 549-559.

23. Jakubowski A, Ambrose C, Parr M, Lincecum JM, Wang MZ, et al. (2005) TWEAK induces liver progenitor cell proliferation. J Clin Invest 115: 2330-2340.

24. Yadavilli S, Mayo LD, Higgins M, Lain S, Hegde V, et al. (2009) Ribosomal protein S3: A multi-functional protein that interacts with both p53 and MDM2 through its KH domain. DNA Repair (Amst) 8: 1215-1224.

25. Waldman T, Kinzler KW, Vogelstein B (1995) p21 is necessary for the p53mediated G1 arrest in human cancer cells. Cancer Res 55: 5187-5190.

26. He G, Siddik ZH, Huang Z, Wang R, Koomen J, et al. (2005) Induction of p21 by p53 following DNA damage inhibits both Cdk4 and Cdk2 activities. Oncogene 24: 2929-2943.

27. Delbridge AR, Valente LJ, Strasser A (2012) The role of the apoptotic machinery in tumor suppression. Cold Spring Harb Perspect Biol 4.

28. Attardi LD (2005) The role of p53-mediated apoptosis as a crucial anti-tumor response to genomic instability: lessons from mouse models. Mutat Res 569: 145-157.

29. Coutts AS, Adams CJ, La Thangue NB (2009) p53 ubiquitination by Mdm2: a never ending tail? DNA Repair (Amst) 8: 483-490.

30. Vousden KH, Prives C (2009) Blinded by the Light: The Growing Complexity of p53. Cell 137: 413-431.

31. Park WH, Seol JG, Kim ES, Hyun JM, Jung CW, et al. (2000) Arsenic trioxidemediated growth inhibition in MC/CAR myeloma cells via cell cycle arrest in association with induction of cyclin-dependent kinase inhibitor, p21, and apoptosis. Cancer Res 60: 3065-3071.

32. Higami Y, Shimokawa I (2000) Apoptosis in the aging process. Cell Tissue Res 301: 125-132.

33. Ruggero D, Pandolfi PP (2003) Does the ribosome translate cancer? Nat Rev Cancer 3: 179-192.

34. Kool M, Koster J, Bunt J, Hasselt NE, Lakeman A (2008) Integrated genomics identifies five medulloblastoma subtypes with distinct genetic profiles, pathway signatures and clinicopathological features. PLoS One, 3e3088.

35. Kim Y, Yoon JW, Xiao X, Dean NM, Monia BP, et al. (2007) Selective downregulation of glioma-associated oncogene 2 inhibits the proliferation of hepatocellular carcinoma cells. Cancer Res 67: 3583-3593.

36. Kim Y, Kim HD, Youn B, Park YG, Kim J (2013) Ribosomal protein S3 is secreted as a homodimer in cancer cells. Biochem Biophys Res Commun 441: 805-808.

37. Nagao-Kitamoto $H$, Setoguchi T, Kitamoto $S$, Nakamura S, Tsuru A, et al (2015) Ribosomal protein S3 regulates GLI2-mediated osteosarcoma invasion. Cancer Lett 356: 855-861. 